\title{
Rapid Microwave Preparation of Thermoelectric TiNiSn and TiCoSb Half-Heusler Compounds
}

Christina S. Birkel, ${ }^{\dagger \ddagger}$ Wolfgang G. Zeier, ${ }^{\text {II, }}{ }^{\dagger}$ Jason E. Douglas, ${ }^{\|, \ddagger}$ Bethany R. Lettiere, ${ }^{\ddagger}$ Carolyn E. Mills, ${ }^{\perp}$ Gareth Seward, ${ }^{\#}$ Alexander Birkel, ${ }^{@}$ Matthew L. Snedaker, ${ }^{\dagger}$ Yichi Zhang, ${ }^{\dagger}$ G. Jeffrey Snyder, ${ }^{\text {II }}$ Tresa M. Pollock, $\|,+$ Ram Seshadri, ${ }^{\dagger, \|, \ddagger}$ and Galen D. Stucky ${ }^{*},, \|$

${ }^{\dagger}$ Department of Chemistry and Biochemistry, University of California, Santa Barbara, California 93106, United States

${ }^{\ddagger}$ Materials Research Laboratory, University of California, Santa Barbara, California 93106, United States

IIMaterials Science, California Institute of Technology, Pasadena, California 91125, United States

${ }^{\S}$ Institut für Anorganische Chemie und Analytische Chemie, Johannes-Gutenberg-Universität, Mainz, DE 55099, Germany

"Materials Department, University of California, Santa Barbara, California 93106, United States

${ }^{\perp}$ Department of Chemical Engineering, University of California, Santa Barbara, California 93106, United States

\# Department of Earth Science, University of California, Santa Barbara, California 93106, United States

${ }^{\circledR}$ Mitsubishi Chemical Center for Advanced Materials, University of California, Santa Barbara, California 93106, United States

\section{Supporting Information}

ABSTRACT: The 18-electron ternary intermetallic systems TiNiSn and $\mathrm{TiCoSb}$ are promising for applications as high-temperature thermoelectrics and comprise earth-abundant, and relatively nontoxic elements. Heusler and half-Heusler compounds are usually prepared by conventional solid state methods involving arc-melting and annealing at high temperatures for an extended period of time. Here, we report an energy-saving preparation route using a domestic microwave oven, reducing the reaction time significantly from more than a week to one minute. A microwave susceptor material rapidly heats the elemental starting materials inside an evacuated quartz tube resulting in near

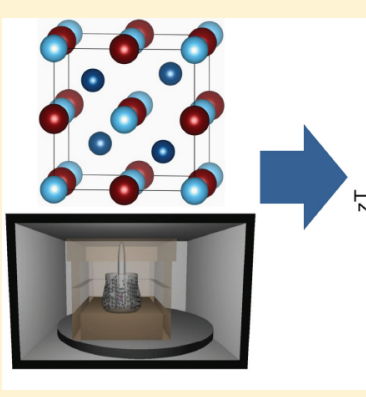
(a) TiNiSn single phase compounds. The initial preparation is followed by a densification step involving hot-pressing, which reduces the amount of secondary phases, as verified by synchrotron X-ray diffraction, leading to the desired half-Heusler compounds, demonstrating that hot-pressing should be treated as part of the preparative process. For TiNiSn, high thermoelectric power factors of $2 \mathrm{~mW} / \mathrm{mK}^{2}$ at temperatures in the 700 to $800 \mathrm{~K}$ range, and $z T$ values of around 0.4 are found, with the microwave-prepared sample displaying somewhat superior properties to conventionally prepared half-Heuslers due to lower thermal conductivity. The TiCoSb sample shows a lower thermoelectric figure of merit when prepared using microwave methods because of a metallic second phase.

KEYWORDS: Heusler, intermetallics, microwave synthesis, thermoelectrics, TiNiSn, TiCoSb

\section{INTRODUCTION}

The 18-electron intermetallic systems $\mathrm{TiNiSn}$ and $\mathrm{TiCoSb}$ are known for their good thermoelectric properties in the intermediate temperature region ${ }^{1}$ and are promising candidates for applications in waste heat recovery for example, due to their earth abundant components. They adopt the half-Heusler MgAgAs crystal structure which consists of three filled interpenetrating face-centered-cubic $(f c c)$ sublattices and one vacant sublattice. ${ }^{2}$ Interestingly, although they each contain three metals, their electronic structures and properties resemble those of semiconducting materials with high thermopowers and reasonably low resistivities, ${ }^{3,4}$ both of which are prerequisites for a high thermoelectric figure of merit $z T$. The semiconducting behavior arises because these 18-electron com- pounds are stuffed zinc-blendes whose electronic structures are closely related to the corresponding III-V semiconductors. ${ }^{5}$

The thermoelectric figure of merit is a materials property directly related to the conversion efficiency and can be described as $z T=\alpha^{2} /(\rho \kappa) \times T$, where $\alpha$ is the Seebeck coefficient, $T$ is the absolute temperature, $\rho$ is the electrical resistivity and $\kappa$ is the thermal conductivity. ${ }^{6}$

Depending on the preparation technique and doping strategy, a variety of thermoelectric properties has been reported for the $\mathrm{TiCoSb}$ and $\mathrm{TiNiSn}$ systems which are the most widely studied compounds among the half-Heusler

Received: April 11, 2012

Revised: $\quad$ May 23, 2012

Published: May 31, 2012 


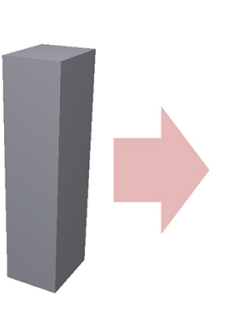

(a)

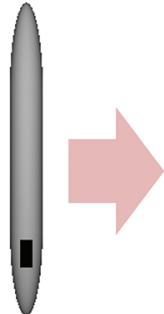

(b)

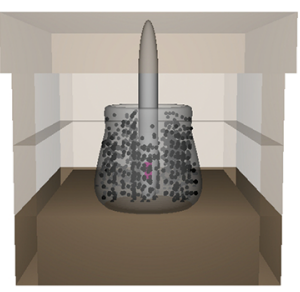

(c)

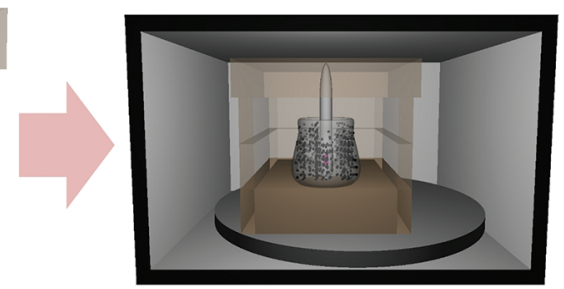

(d)

Figure 1. Schematic of the microwave heating procedure.

materials. TiCoSb is known to exhibit a relatively low thermoelectric figure of merit of below 0.1 at high temperatures. ${ }^{7}$ This can be improved through different alloying and doping techniques and Xie et al. and Qin et al. reported maximum $z T$ values of 0.2 and 0.7 , respectively. ${ }^{8,9}$ Similar strategies have been employed in the case of TiNiSn, which exhibits a higher figure of merit compared to $\mathrm{TiCoSb}$. Bhattacharya et al. investigated the effect of Sb-doping on the low temperature electronic ${ }^{10}$ and thermal ${ }^{11,12}$ transport properties and showed an enhancement of the figure of merit compared to undoped TiNiSn. Additional doping and its effect on the high temperature thermoelectric properties of $\mathrm{TiNiSn}$ have been examined by Culp et al. for example. They studied $\mathrm{Zr}$ - and Hf-doping on the Ti site combined with Sb-doping on the $\mathrm{Sn}$ site and reported a maximum $z T$ of 0.8 at around 1000 $\mathrm{K}^{13}$

Conventionally, intermetallics are prepared via solid state methods where metals are alloyed under inert conditions either by arc-melting or using an induction furnace. This process is usually followed by an additional annealing step in evacuated quartz tubes for several days. ${ }^{14-18} \mathrm{~A}$ possible energy-saving strategy to access these intermetallic materials is by the use of microwave ovens. Two major advantages of this technique are the possibility of using inexpensive commercial microwave reactors and the rapid time- and energy-efficient preparation of the desired compounds. Microwave-assisted solid state reactions have been used since the $1980 \mathrm{~s}^{19-21}$ in order to prepare a variety of materials, such as metallic ${ }^{22,23}$ and ceramic compounds, ${ }^{24-27}$ and even thermoelectric materials. ${ }^{28}$ This technique relies on dielectric heating and thus the ability of a material to couple to the microwave radiation, which is described as its dielectric loss tangent. If this is too low, a susceptor material (e.g., $\mathrm{C}, \mathrm{SiC}, \mathrm{CuO}$ ) can be used which interacts efficiently with the microwave radiation thereby heating the precursors. ${ }^{29}$ This direct heating can be realized by putting the starting material in contact with or in close proximity to the susceptor material. Once the reactants reach a certain temperature the dielectric loss tangent increases sufficiently to enable additional heating within the material itself.

Compared to the conventional heating mechanisms using a furnace, microwave heating is believed to lead to enhanced diffusion followed by rapid grain growth. ${ }^{30}$ Additionally, in the case of TiNiSn and $\mathrm{TiCoSb}$, the three metals have different melting points, that is $\mathrm{Sn}$ and $\mathrm{Sb}$ melt at much lower temperatures than $\mathrm{Ni}, \mathrm{Co}$, and $\mathrm{Ti}$. This typically results in issues in terms of the microstructure and phase purity of the obtained intermetallic compounds. For example, Kimura et al. investigated (Ti/Zr)NiSn, (Ti/Hf) NiSn and ( $\mathrm{Zr} / \mathrm{Hf}) \mathrm{NiSn}$ and pointed out that the different Ti-rich or -poor areas within those samples have a big effect on their thermoelectric properties. ${ }^{31}$

Here, we describe the optimization of an energy-saving microwave preparation of TiNiSn and TiCoSb. The success of this new approach to the synthesis of intermetallic systems is demonstrated by a detailed comparison of these compounds and their properties with conventionally prepared analogues.

The entire process not only involves the preparation of the initial materials but also their densification by hot-pressing, which allows for thermoelectric characterization. Since the densification step also includes a heating period, it must be considered as part of the preparation process, and we demonstrate how it affects particularly the microwave prepared samples. Since thermoelectric properties of these half-Heusler compounds are sensitive to deviations in the composition and microstructure, we carefully investigate the obtained samples along the entire processing path including the ball-milled compounds as well as the hot-pressed samples used for thermoelectric measurements. In addition, we measure the transport properties during multiple heating and cooling cycles to address the structural stability of the two different halfHeusler systems.

\section{EXPERIMENTAL DETAILS}

In the course of this study, four different samples were prepared. Two samples were synthesized by the more conventional solid state method through arc-melting and annealing while a novel rapid microwave preparation was used to prepare the same compounds in a more energy efficient way. The 18-electron systems, TiNiSn and TiCoSb, were chosen to demonstrate the potential of rapid microwave heating as well as the influence of hot-pressing on the materials properties. The following metal powders were used as starting materials: $\mathrm{Ni}$ (99.99\%, Sigma-Aldrich), Ti (99.7\%, Sigma-Aldrich), Sn (200 mesh, Matheson), Co (99.9\%, Sigma-Aldrich), and Sb (99.5\%, Alfa Aesar).

Preparation. For the conventionally prepared samples, stoichiometric amounts of metal powders (stored in an argon filled glovebox) were cold-pressed into cylindrical pellets.The shiny metallic pellets were arc melted once using a Buehler MAM1 instrument to form a gray button. These buttons were wrapped in tantalum foil (SigmaAldrich) and sealed into evacuated quartz tubes, which were then annealed vertically at $1173 \mathrm{~K}$ for $24 \mathrm{~h}$ and at $1123 \mathrm{~K}$ for an additional 8 days. These samples will be referred to as arc-melted (AM) samples.

The microwave heating procedure followed the prior work of Ramesh et al. ${ }^{28}$ The general process is shown in Figure 1.

Stoichiometric amounts of metal powders were cold-pressed into bar shaped pellets $(9 \mathrm{~mm} \times 3 \mathrm{~mm} \times 3 \mathrm{~mm}$ ) (Figure 1a) and directly sealed into evacuated quartz tubes (Figure $1 \mathrm{~b}$ ). The tubes were placed into a crucible filled with $3.6 \mathrm{~g}$ of granular carbon (Darco 12 mesh to 20 mesh, Sigma-Aldrich) which acts as the microwave susceptor material. In order to minimize heat loss it was surrounded by a housing 
of high temperature alumina insulation foam (Figure 1c) and the whole setup was placed into a commercial microwave reactor (LG Goldstar) with a rotating plate (Figure 1d). The reactions were allowed to run at $100 \%$ power $(700 \mathrm{~W})$ for $1 \mathrm{~min}$. After a couple of seconds a purplish plasma could be observed through the gaps in the housing surrounding the quartz tube which was followed by bright orange glow when the reaction was completed. The setup was allowed to cool before it was removed from the microwave. These samples are referred to as microwave (MW) samples. Note that no comparable reaction takes place if no susceptor material is used which differs from the experiments reported by Landry et al. ${ }^{21}$

Processing. AM samples were found to maintain their button form and the MW samples to take the form of the quartz tube during preparation. After the initial preparation all compounds were processed in the same way. The samples were crushed using a percussion mortar and ground into pieces no larger than about $5 \mathrm{~mm}$ using an alumina mortar. In order to obtain a fine powder, the small pieces were ball milled with acetone in a WC container for $10 \mathrm{~min}$ using a SPEX $8000 \mathrm{M}$ Mixer/Mill. The dispersion was then allowed to dry in a crystallization dish and the powder was filled into a BN coated graphite die (POCO, EDM-3, Saturn Industries) with a diameter of about $11 \mathrm{~mm}$. All powders were hot-pressed with the same pressing profile (for details see Supporting Information) with a pressure of about $84 \mathrm{MPa}$ at $1173 \mathrm{~K}$ for $60 \mathrm{~min}$ using an OXYGON Mini hot press.

After they were cooled to room temperature, the cylindrical pellets were removed from the die and polished with $\mathrm{SiC}$ grinding paper (different roughnesses) to clean the surfaces from all remaining BN powder.

For measurement of the electronic properties bar-shaped pieces with the approximate dimensions $8 \mathrm{~mm} \times 2 \mathrm{~mm} \times 1.5 \mathrm{~mm}$ were cut using a low-speed saw (Allied Inc.) with a diamond wafering blade.

The remaining pieces were polished further to obtain a smooth surface for additional characterizations. Samples were fixed into an epoxy (Buehler Epomet) by hot compression mounting and polished using the following procedure. The samples were ground using silicon carbide abrasive paper and then polished to $0.25 \mu \mathrm{m}$ using colloidal diamond on cloth. All samples were then sonicated and etched using a solution of $35 \%$ nitric acid $/ 30 \%$ water $/ 30 \%$ acetic acid $/ 5 \%$ hydrofluoric acid.

Characterization. Powder X-ray diffraction data were obtained on a Philips X'Pert with $\mathrm{CuK} \alpha$ radiation. In addition, high resolution synchrotron powder diffraction data were collected at beamline 11-BM at the Advanced Photon Source (APS), Argonne National Laboratory using a wavelength of $\lambda=0.41315 \AA$. Rietveld analyses were carried out using the XND Rietveld code. ${ }^{32}$

Field-emission scanning electron microscopy was performed on a FEI XL40 Sirion FEG microscope with an Oxford Inca X-ray system attached for chemical analysis. In addition, a FEI XL30 microscope equipped with a backscattered electron detector (BSE) was used to maximize the contrast between phases with similar atomic composition. After polishing the mounted samples (in epoxy), they were surrounded with silver paste to separate the sample from the nonconductive material in order to prevent the sample from charging during imaging. Secondary and backscattered electron images were collected with an acceleration voltage of $5 \mathrm{keV}$ and 15 or $20 \mathrm{keV}$, respectively. Electron microprobe analyses were conducted using a Cameca SX100 equipped with 5 wavelength dispersive spectrometers (WDS). Probe image and probe for EPMA (Probe software Inc.) were used for acquisition of X-ray intensity maps and quantitative data respectively (see Supporting Information for analytical conditions). To visualize chemical compositional differences within the X-ray map data, individual element X-ray intensity channels were combined into three channels of a color image to create a false color composite image of X-ray intensity for all elements. Seebeck coefficient and electrical resistivity in the temperature region between 300 and $875 \mathrm{~K}$ were measured simultaneously on bar-shaped samples using a ZEM-3 Ulvac instrument. Thermal diffusivity was measured on discs using a Netzsch laser flash diffusivity instrument (LFA 457), samples were coated with a thin layer of graphite to minimize errors in the emissivity. The data were analyzed using a Cowan model with pulse correction. Heat capacity $\left(C_{\mathrm{p}}\right)$ made use of the Dulong-Petit estimate, $\left(C_{\mathrm{p}}=3 R\right)$ and theoretical densities were calculated from measured mass-by-volume ratios. Densities were found to be between $91 \%$ and $95 \%$ of the theoretical density.

\section{RESULTS AND DISCUSSION}

Phase and Microstructure Characterization. Powder Xray diffraction data of the $\mathrm{TiNiSn}$ and $\mathrm{TiCoSb}$ samples are shown in Figure 2 and Figure 3, respectively. To identify the

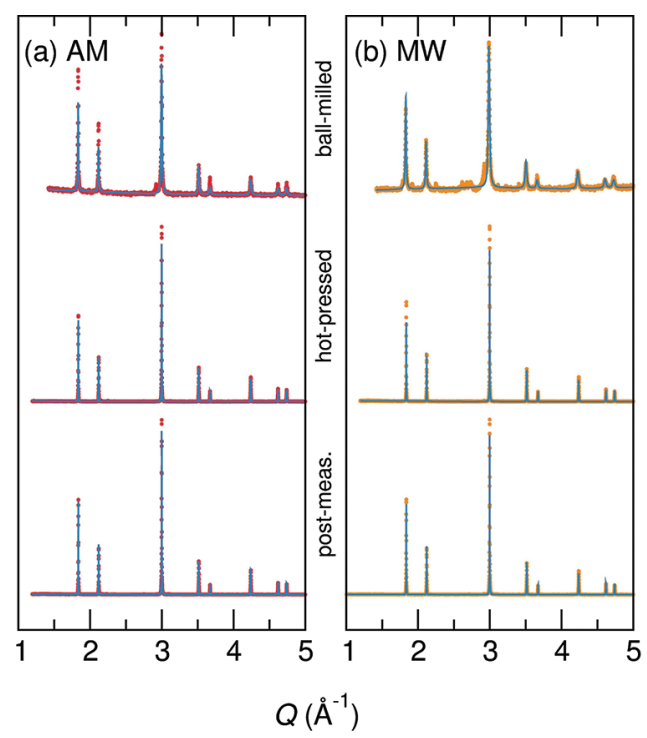

Figure 2. X-ray powder diffraction data of TiNiSn samples obtained during the preparative process: (a) arc-melted and annealed sample, (b) microwave-prepared sample. Circles represent data and solid lines the calculated Rietveld fits of the half-Heusler crystal structure. $Q=$ $2 \pi / d$ represents the momentum transfer function; $Q=4 \pi \sin (\vartheta / \lambda)$.

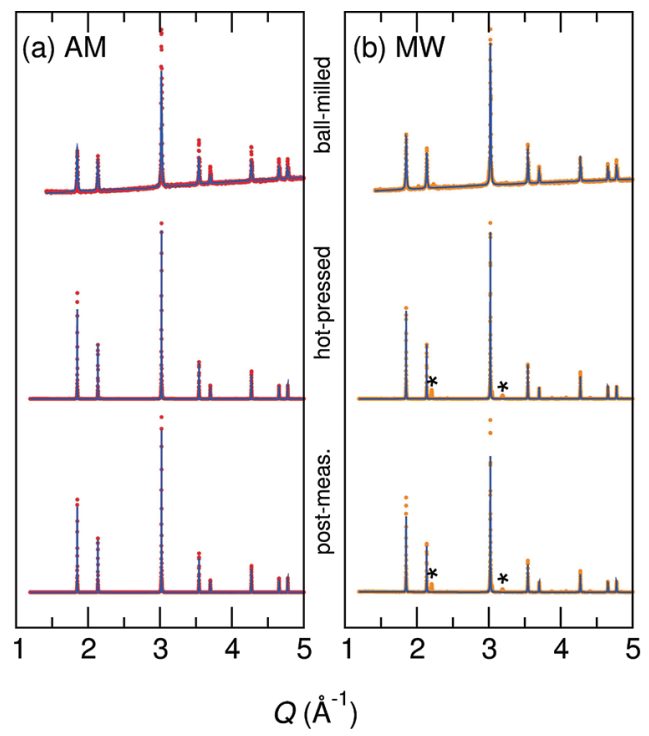

Figure 3. X-ray powder diffraction data of TiCoSb samples, obtained during the preparative process: (a) arc-melted and annealed sample, (b) microwave-prepared sample. Circles represent data and solid lines the calculated Rietveld fits of the half-Heusler crystal structure. The stars indicate the main peaks of the impurity CoSb phase in the MW sample. 
Table 1. Refined Parameters (Cell Parameter, $a$, Residual Factor, $R_{\text {Bragg, }}$ and Crystallite Size, CS) of Hot-Pressed (HP) and PostMeasurement (PM) Samples

\begin{tabular}{|c|c|c|c|c|c|c|c|}
\hline \multicolumn{4}{|c|}{ TiNiSn } & \multicolumn{4}{|c|}{$\mathrm{TiCoSb}$} \\
\hline method/process & $a(\AA)$ & $R_{\text {Bragg }}(\%)$ & CS $(\mathrm{nm})$ & method/process & $a(\AA)$ & $R_{\text {Bragg }}(\%)$ & CS (nm) \\
\hline $\mathrm{AM} / \mathrm{HP}$ & $5.92987(2)$ & 7.3 & 110 & $\mathrm{AM} / \mathrm{HP}$ & $5.88434(1)$ & 4.5 & 220 \\
\hline $\mathrm{AM} / \mathrm{PM}$ & $5.92890(1)$ & 3.6 & 130 & $\mathrm{AM} / \mathrm{PM}$ & $5.88438(1)$ & 2.5 & 220 \\
\hline $\mathrm{MW} / \mathrm{HP}$ & $5.92952(2)$ & 7.0 & 100 & $\mathrm{MW} / \mathrm{HP}$ & $5.8836(2)$ & 6.7 & 180 \\
\hline $\mathrm{MW} / \mathrm{PM}$ & $5.92927(2)$ & 4.4 & 130 & $\mathrm{MW} / \mathrm{PM}$ & $5.8836(2)$ & 7.5 & 180 \\
\hline
\end{tabular}

crystalline phases formed during the complete preparation and processing procedures, data were obtained on the ball-milled and hot-pressed samples, as well as on the materials after the ZEM measurement. Among these data sets, synchrotron X-ray radiation was used for the analyses of the hot-pressed and postmeasurement samples.

Table 1 summarizes the lattice constant $a$, residual factors

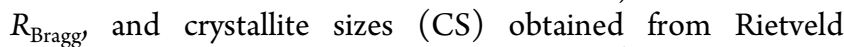
refinements of the synchrotron XRD data (hot-pressed and postmeasurement samples). The refined lattice constants of TiNiSn and TiCoSb match those reported in the literature ${ }^{2,14}$ and only very small deviations are observed for the individual samples. In the case of the TiNiSn samples, the refined residual factors of the postmeasurement samples are smaller while their crystallite sizes are larger than the corresponding ones of the hot-pressed samples. This could be attributed to slightly increased crystallinity due to the high temperature measurement. This effect is not observed clearly for the $\mathrm{TiCoSb}$ samples.

All data could be refined using the lattice parameters of the half-Heusler structure crystallizing in space group $\bar{F} \overline{4} 3 \mathrm{~m}$ (see also inset in Figure 4). Ball-milled TiNiSn compounds show

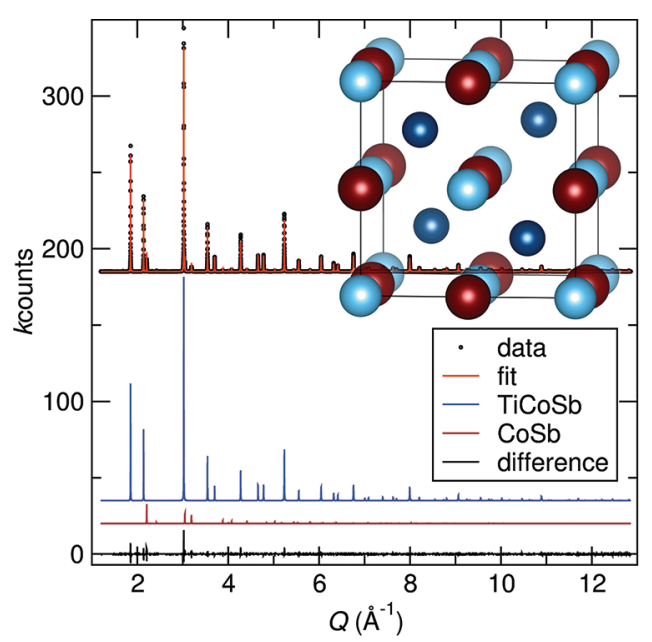

Figure 4. Synchrotron X-ray powder diffraction data of the hotpressed TiCoSb sample (MW) including a two-phase Rietveld refinement corresponding to the half-Heusler and $\mathrm{CoSb}$ phases. Circles represent data, colored solid lines the calculated fits and the black solid line the difference profile. The inset shows the half-Heusler crystal structure.

some impurity peaks corresponding to full-Heusler $\mathrm{TiNi}_{2} \mathrm{Sn}$ and an additional binary phase $\left(\mathrm{Sn}_{3} \mathrm{Ti}_{5}\right)$ in the case of the microwave prepared sample. However, synchrotron XRD data of the hot-pressed samples allow an essentially clean refinement of single phase half-Heusler TiNiSn. This clearly demonstrates the influence of the hot-pressing step after the initial reaction, that is the elimination of the largest part of additional impurity phases. In the case of $\mathrm{TiCoSb}$, the initial ball-milled compounds are nearly free of secondary phases. Synchrotron XRD data of the conventionally prepared compound show a single phase half-Heusler structure. On the other hand, hot-pressing of the microwave prepared sample did not result in a clean product since peaks of $\mathrm{CoSb}$ were identified. The fraction of the $\mathrm{CoSb}$ impurity phase was calculated to be about $10 \mathrm{~mol} \%$. Figure 4 shows the two-phase refinement of the synchrotron XRD data of the hot-pressed microwave TiCoSb sample including an inset of the half-Heusler crystal structure.

The quality of the hot-pressed pellets has been confirmed by electron micrographs of their polished surfaces which exhibit some small voids but overall a sufficient density (Supporting Information Figure SI-1). Additional images recorded in backscatter mode (Supporting Information Figure SI-2) show higher and lower backscatter-intensity areas suggesting inhomogeneity in the samples in terms of elemental composition. To elucidate possible different phases, electron microprobe X-ray intensity maps were collected from each of the four samples (see Figure 5).
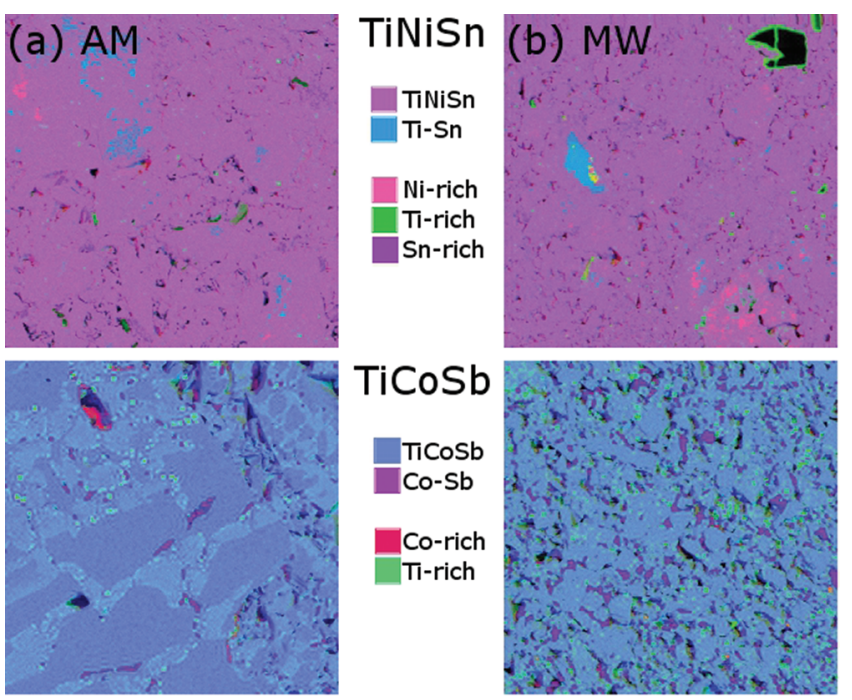

Figure 5. X-ray intensity maps of the surface of the different TiNiSn and TiCoSb samples: (a) arc-melted and annealed samples and (b) microwave-prepared samples. Different colors correspond to regions of different elemental compositions. Images are $255 \mu \mathrm{m} \times 255 \mu \mathrm{m}$.

The combination of qualitative X-ray intensity map data and EDX analyses (Supporting Information Figure SI-3) confirm that the bulk of the mapped area in all samples is the ternary half-Heusler phase. This was further confirmed by WDS X-ray analysis (not shown here). The largest deviation from the 1:1:1 half-Heusler composition is about $1 \%$, when scaled to total halfHeusler composition, rather than individual elements. How- 
ever, the composite X-ray maps also confirm the presence and location of additional areas with different compositions. In the case of the TiNiSn samples, there are Ni-deficient regions of binary $\mathrm{Ti}_{6} \mathrm{Sn}_{5}$ (light blue) and also areas where each of the elements is most dominant. Similarly, in the TiCoSb samples, Ti-deficient segregated regions containing the $\mathrm{CoSb}$ phase are present with additional Co- and Ti-rich areas. While the AM and MW TiNiSn samples show almost the same bulk compositions, the microwave prepared $\mathrm{TiCoSb}$ sample exhibits a greater area/volume fraction of $\mathrm{CoSb}$ regions than the conventionally prepared sample. This relationship can also be observed in the synchrotron XRD data (Figure 4).

Except for the aforementioned $\mathrm{CoSb}$ regions, none of the other phases can clearly be detected using X-ray diffraction. However, enlarging the low order area of the XRD data of the hot-pressed sample, several peaks of very low intensity can be found (see Figure 6). For the TiNiSn samples, it is troublesome

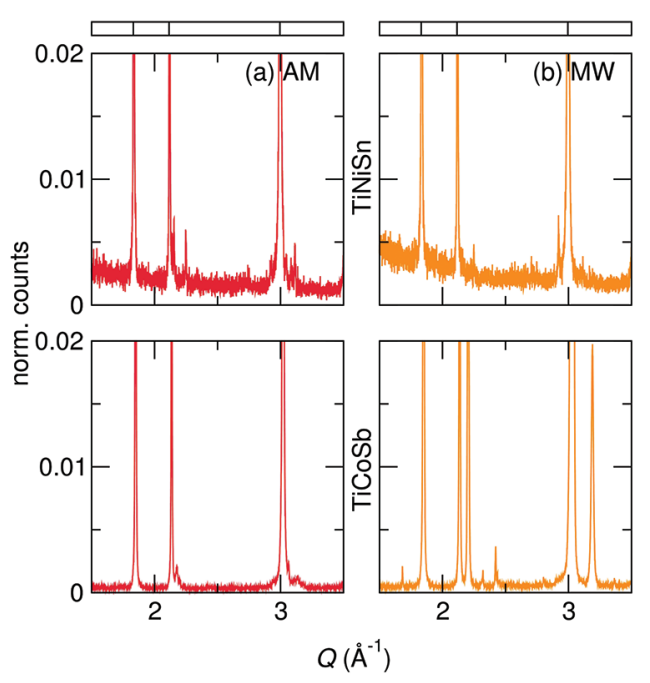

Figure 6. Low-order XRD data of the different hot-pressed samples showing the minor impurity peaks. Only the MW TiCoSb sample shows a more significant amount of CoSb impurity ( $10 \mathrm{~mol} \%)$. Tick marks indicate the half-Heusler peak positions.

to identify those phases due to a low signal-to-noise ratio which is a result of X-ray absorption caused by tin. However, it can be assumed that those peaks correspond to the phases found by microprobe analysis. In the case of the TiCoSb samples, the $\mathrm{CoSb}$ impurity can clearly be observed in Figure 6 for the MW sample while the AM sample exhibits very low intensity peaks that also correspond to this phase. This is not surprising since it is also detected by microprobe analysis and even expected due to the decomposition of $\mathrm{TiCoSb}$ at temperatures above $750 \mathrm{~K}$.

Thermoelectric Characterization. Room-temperature thermoelectric data of the four different samples are summarized in Table 2.

To test the performance and stability of the materials, the thermoelectric properties were measured during multiple heating and cooling cycles. For the electronic properties, data were recorded for three successive cycles while one heating and one cooling set were recorded in the case of the thermal diffusivity measurements. Data were collected up to a maximum temperature of about $880 \mathrm{~K}$ for the TiNiSn samples and up to about $680 \mathrm{~K}$ for the $\mathrm{TiCoSb}$ samples since a decomposition occurs at $\sim 680 \mathrm{~K}^{33}$

Electronic Transport Properties. The electronic properties, including these for all samples during three heating and cooling cycles are shown in the Supporting Information (Figure SI-4). To simplify all plots only data obtained during the first heating and cooling segments are presented. In general, especially in the case of the electronic properties, there is a difference in the data measured during heating and cooling which becomes less pronounced in the course of additional cycles so that more stable values are achieved. For all samples, the resistivity and the absolute value of the Seebeck coefficient decrease, in some cases quite significantly indicating that the materials become "more metallic" during the measurement. It can be assumed that a phase separation takes place during the measurement, introducing charge carriers as a result of a slightly non stoichiometric matrix, with higher carrier concentrations leading to the change in the Seebeck coefficient and the resistivity. This is supported by the microprobe images showing different metal-rich areas that become more pronounced during the first heating segment. As noted above, a change in the phase composition could not be verified by the synchrotron XRD data before and after the measurement (see Figures 2 and 3).

The temperature dependence of the electrical resistivity, Seebeck coefficient and resulting power factor $\alpha^{2} / \rho$ for all four samples is shown in Figure 7. Open and filled symbols are used for data points obtained during heating and cooling, respectively. Solid and broken lines show polynomial fits which are used to calculate the thermoelectric figure of merit.

Room-temperature resistivity values of the TiNiSn samples are comparable to those reported by Bhattacharya et al., for example in reference. ${ }^{11}$ Both TiNiSn compounds show typical small-bandgap semiconducting behavior, that is decreasing resistivity with increasing temperature (Figure 7a) with a higher resistivity in the MW sample.

Different Seebeck coefficients for TiNiSn have been reported in the last decades ranging from $-150 \mu \mathrm{V} / \mathrm{K}^{11}$ to $-250 \mu \mathrm{V} / \mathrm{K}^{10}$ and even reaching high negative values of around $-300 \mu \mathrm{V} /$ $\mathrm{K} .{ }^{17,34} \mathrm{We}$ find room temperature Seebeck coefficients of -140 $\mu \mathrm{V} / \mathrm{K}$ and $-250 \mu \mathrm{V} / \mathrm{K}$ for the two TiNiSn samples which are comparable to some of the literature reports. Seebeck coefficients are negative indicating that electrons are the major carrier type in these systems (n-type). The roomtemperature Seebeck coefficient of the MW sample exceeds that of the AM sample with a difference of about $100 \mu \mathrm{V} / \mathrm{K}$ indicating a lower carrier concentration, which is in accordance with the higher electrical resistivity in these samples.

The Seebeck coefficients for both TiNiSn samples exhibit two different regions of behavior. First $\alpha$ becomes more

Table 2. Room-Temperature Thermoelectric Data and Relative Densities of AM and MW TiNiSn and TiCoSb

$\begin{array}{cccccccc}\text { sample } & \rho(\mathrm{m} \Omega \mathrm{cm}) & \alpha(\mu \mathrm{V} / \mathrm{K}) & \kappa(\mathrm{W} / \mathrm{m} \cdot \mathrm{K}) & \kappa_{\mathrm{el}}(\mathrm{W} / \mathrm{m} \cdot \mathrm{K}) & \kappa-\kappa_{\text {el }}(\mathrm{W} / \mathrm{m} \cdot \mathrm{K}) & L\left(\mathrm{~W} \Omega / \mathrm{K}^{2}\right) & \text { rel. } d(\%) \\ \text { AM TiNiSn } & 6.7 & -140 & 4.8 & 0.08 & 4.7 & 1.8 & 96 \\ \text { MW TiNiSn } & 9.7 & -255 & 4.6 & 0.05 & 4.6 & 1.7 & 93 \\ \text { AM TiCoSb } & 141.4 & -297 & 11.5 & 0.003 & 11.5 & 1.5 & 95 \\ \text { MW TiCoSb } & 12.7 & -10 & 12.0 & 0.06 & 11.9 & 2.4\end{array}$


(a) TiNiSn
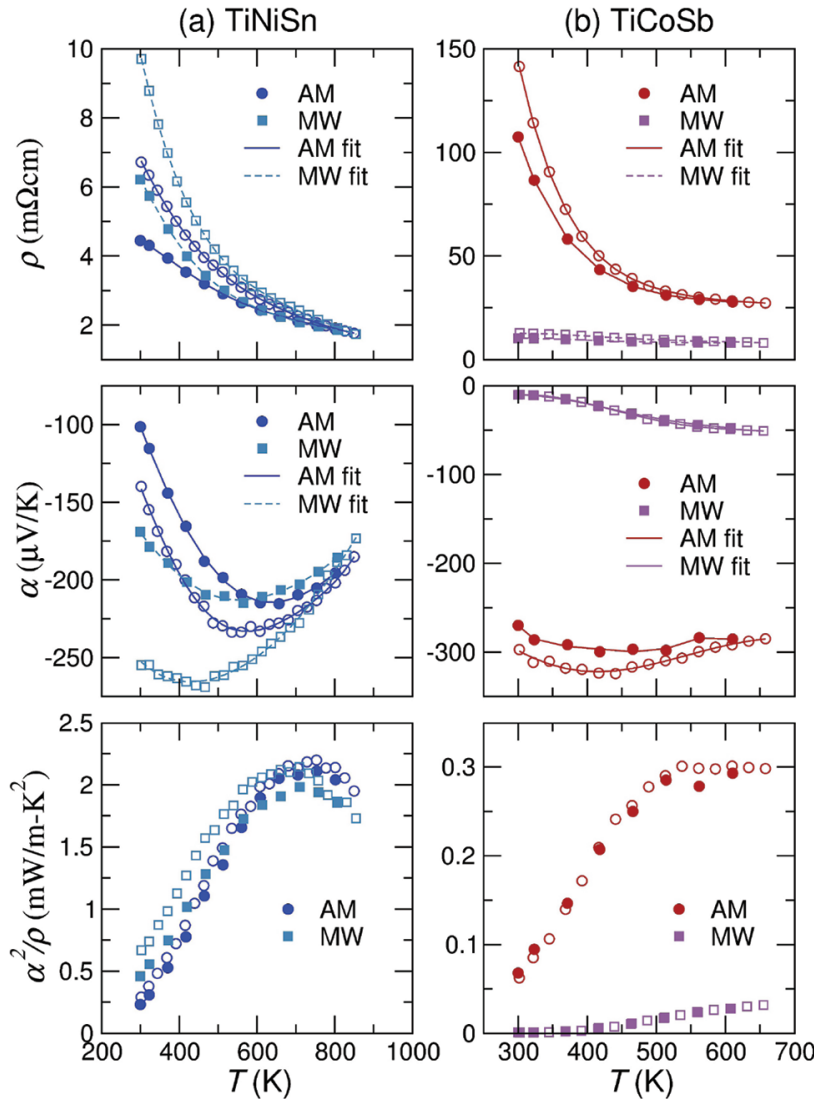

Figure 7. Electronic properties (resistivity, Seebeck coefficient, and power factor) of the differently prepared $\mathrm{TiNiSn}$ (a) and $\mathrm{TiCoSb}$ (b) samples. Open and filled symbols correspond to data obtained during heating and cooling, respectively. Solid lines are fifth degree polynomial fits. Note that MW-TiCoSb contains about $10 \mathrm{~mol} \%$ of metallic $\mathrm{CoSb}$.

negative ( $|\alpha|$ increasing) reaching a maximum value before $|\alpha|$ decreases with increasing temperature. This change in Seebeck coefficient can be explained by the excitation of electron-hole pairs across the energy gap, which means that the opposite contribution to $\alpha$ reduces the absolute value of $\alpha$. Therefore, the existence of this phenomenon is dependent on the band gap of the material.

While both TiNiSn samples behave in a similar manner in terms of their electronic properties, the TiCoSb samples show a significant difference in resistivity between the AM and the MW sample. While the AM sample also exhibits typical semiconducting behavior, the microwave prepared sample shows a more metallic behavior with a much lower resistivity and different temperature behavior. The temperature dependence and absolute values of the Seebeck coefficients show the same difference between the AM and the MW sample. The Seebeck coefficient of $\mathrm{AM} \mathrm{TiCoSb}$ is large and negative and with an increasing $|\alpha|$ before it decreases at higher temperatures. The Seebeck coefficient therefore behaves in a similar way to that of the TiNiSn compounds. The MW sample, however, exhibits an order of magnitude lower absolute Seebeck coeffcient that increases with increasing temperature. This can be explained by the presence of metallic $\mathrm{CoSb}$ in the $\mathrm{MW} \mathrm{TiCoSb}$ pellet, as observed in the synchrotron XRD data. Although the amount of $\mathrm{CoSb}$ is not very high ( $\sim \mathrm{wt} \%)$, it has a significant effect on the electronic transport property of the overall sample possibly due to a much higher carrier concentration compared to the

AM sample. Room-temperature resistivity and Seebeck coefficient of AM TiCoSb compare well to values reported in the literature. $^{35}$

The power factor of the four different samples are also presented in Figure 7. Both TiNiSn compounds exhibit rather high power factors of around $2 \mathrm{~mW} / \mathrm{mK}^{2}$ at about $700-800 \mathrm{~K}$. In contrast, because of higher resistivity, the power factor of $\mathrm{AM} \mathrm{TiCoSb}$ only reaches $0.3 \mathrm{~mW} / \mathrm{mK}^{2}$ at about $550 \mathrm{~K}$. Despite a lower resistivity, the power factor of the MW sample is another order of magnitude lower since the absolute value of Seebeck coefficient has dropped significantly in this material due to $\mathrm{CoSb}$ impurities. The power factors of the TiNiSn samples pass through a maximum while those of the $\mathrm{TiCoSb}$ compounds do not reach their maximum value before their decomposition temperature is reached.

Thermal Transport Properties. Thermal diffusivity of the materials was measured from room temperature up to 870 and $670 \mathrm{~K}$ and the thermal conductivity was calculated using $\kappa=$ $D d C_{p}$, where $D$ is the thermal diffusivity, $d$ is the density, and $C_{p}$ is the specific heat. The Dulong-Petit approximation is used for the specific heat capacity, which is likely to result in an about $10 \%$ underestimation of the thermal conductivity at high temperatures. ${ }^{36}$ The temperature dependent thermal conductivity data for all four samples are presented in Figure 8. For the thermal conductivity, data during heating and cooling are shown and both curves lie on top of each other.

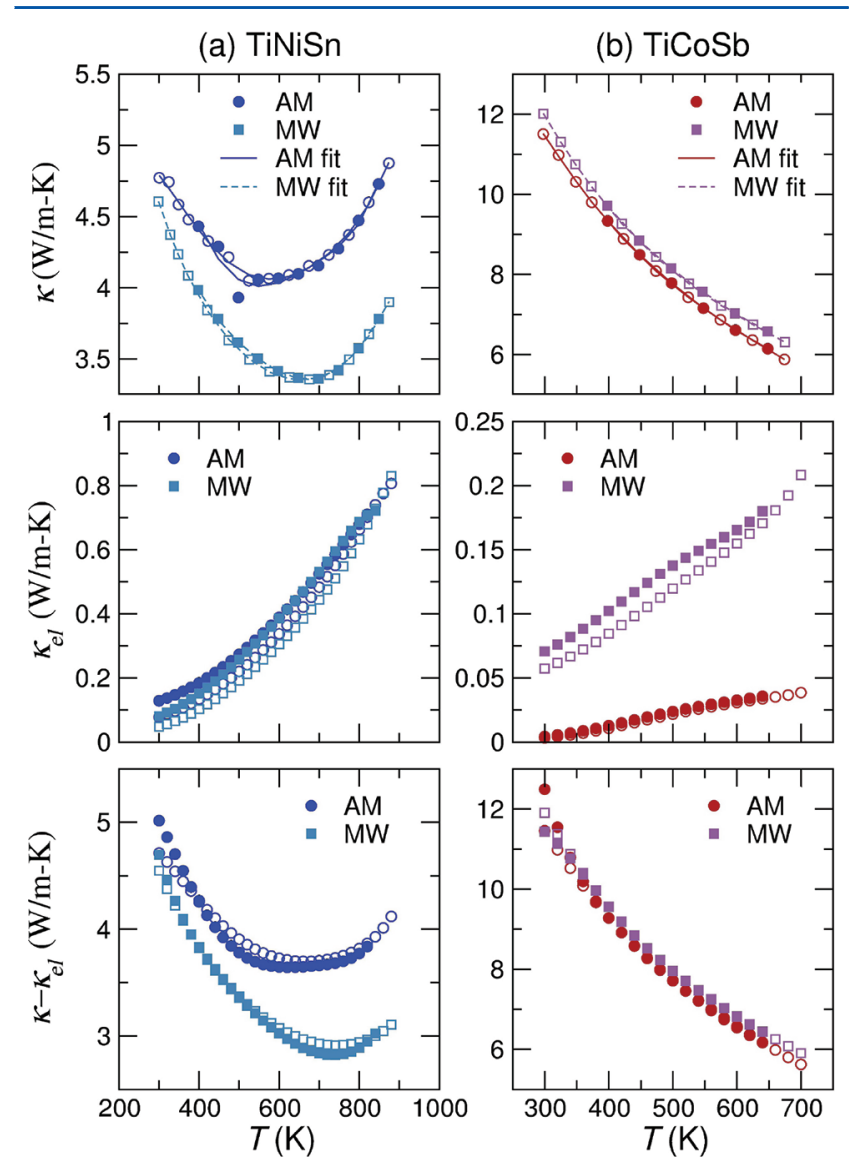

Figure 8. Thermal conductivity and its electronic and lattice contribution of the (a) TiNiSn and (b) TiCoSb samples. Open and filled symbols correspond to data obtained during heating and cooling, respectively. Solid lines are fifth degree polynomial fits. Note that MW$\mathrm{TiCoSb}$ contains about $10 \mathrm{~mol} \%$ of metallic CoSb. 
The total thermal conductivity is the sum of electronic, lattice and bipolar contributions $\left(\kappa=\kappa_{\mathrm{el}}+\kappa_{\mathrm{lat}}+\kappa_{\mathrm{bipol}}\right)$. The Wiedemann-Franz law describes the relation between the electronic resistivity and the electronic part of the thermal conductivity: $\kappa_{\mathrm{el}}=L T / \rho$, where $L$ is the Lorenz number, $T$ is the temperature, and $\rho$ the electrical resistivity. Temperaturedependent Lorenz numbers were calculated following the procedure reported by May et al. ${ }^{37}$ Except for MW TiCoSb, all Lorenz numbers are significantly lower than the value for the free electron model $\left(2.44 \mathrm{~W} \Omega / \mathrm{K}^{2}\right.$, see Table 2$)$.

The temperature dependent data of $\kappa_{e l}$ and $\kappa-\kappa_{e l}$ are also presented in Figure 8. The electronic thermal conductivities increase with increasing temperature which is in agreement with the decrease in electronic resistivity due to the semiconducting nature of the samples. The thermal conductivity of the TiNiSn samples shows a significant bipolar contribution which is visible as an upturn in $\kappa$ above a certain temperature. This phenomenon is not observed in the case of the TiCoSb samples (larger bandgap) since it is a result of the smaller band gap of TiNiSn.

In all cases, the major contribution to the total thermal conductivity is the lattice (for the TiNiSn samples including the bipolar) thermal conductivity. Within the TiNiSn system, the values of $\kappa_{\mathrm{el}}$ are almost the same for the AM and the MW samples while $\kappa-\kappa_{\text {el }}$ differs significantly, especially at high temperatures where the difference is about $1 \mathrm{~W} / \mathrm{mK}$. At room temperature, $\kappa$ of the MW sample is slightly smaller than the one of the AM sample. In the TiCoSb system, $\kappa_{\mathrm{el}}$ of the MW sample is about an order of magnitude higher than the one of $\mathrm{AM} \mathrm{TiCoSb}$ (in accordance with a much lower resistivity in this sample), the lattice contribution to the thermal conductivity is almost the same. The reason for the lower $\kappa-\kappa_{\mathrm{el}}$ at room temperature in the MW TiNiSn sample compared to the AM sample could be the slightly lower density of the hot-pressed sample (see Table 2 ) or possibly smaller grain sizes as a result of the microwave preparation method. While the AM samples are arc melted and then annealed for 10 days, the MW samples are only subject to a very short heating period inside the microwave $(1 \mathrm{~min})$ which does not allow for comparable grain growth. These smaller grains could be responsible for more grain boundary scattering of the phonons followed by a lower lattice contribution to the thermal conductivity. At lower temperatures $\kappa-\kappa_{\mathrm{el}}$ exhibits a temperature dependence associated with phonon-phonon Umklapp scattering. However, with increasing temperature $\kappa-\kappa_{\mathrm{el}}$ of the AM sample deviates significantly from the MW sample, indicating a stronger bipolar contribution.

Thermoelectric Figure of Merit. The resulting figures of merit for all samples are shown in Figure 9.

In the case of the TiNiSn system, $z T$ increases with increasing temperatures and reaches maximum values of 0.35 and 0.45 at around 780 and $740 \mathrm{~K}$ for the AM and the MW sample, respectively. These numbers are comparable to those reported in the literature whereas $z T$ for the $M W$ sample is higher than that of the AM sample because of a lower thermal conductivity.

The figures of merit for the TiCoSb samples also increase with increasing temperatures but do not reach their maximum value within this temperature range. Again, this is due to the larger band gap of $\mathrm{TiCoSb}^{38}$ which does not allow the thermal activation of minority carriers at temperatures below the decomposition temperature of the material. Compared to the highest $z T$ of the TiNiSn samples, the maximum $z T$ of about
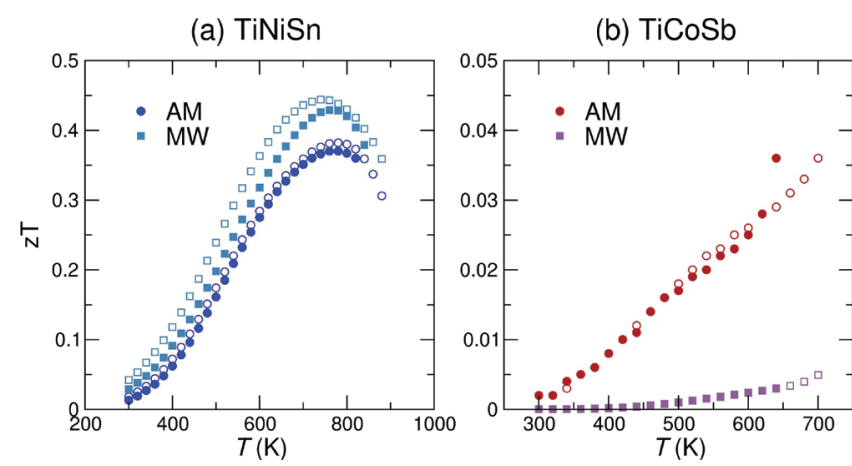

Figure 9. Thermoelectric figure of merit of the differently prepared TiNiSn (a) and TiCoSb (b) samples. Open and filled symbols correspond to data obtained during heating and cooling, respectively. Note that MW-TiCoSb contains about $10 \mathrm{~mol} \%$ of metallic CoSb.

0.04 and 0.004 of the AM and the MW TiCoSb are about one and 2 orders of magnitude lower, respectively, due to a not optimized doping level.

\section{CONCLUSIONS}

We have presented an energy-saving preparative route using a commercial microwave oven to access the half-Heusler compounds TiNiSn and TiCoSb. Starting from compacted metal powders in sealed quartz tubes, this method allows the reaction time to be reduced from over a week to as little as 1 min. The initial preparation is followed by a compaction step using a hot press which not only densifies the ball-milled materials but also reduces impurity phases and results in single phase materials in all cases except for the microwave prepared $\mathrm{TiCoSb}$. This demonstrates the influence of hot-pressing, which clearly should be considered an additional preparative step. Therefore, we follow the entire process through means of synchrotron powder X-ray diffraction, and study the hotpressed materials which are characterized in terms of their thermoelectric properties very carefully. Although the XRD data show predominantly single-phase half-Heusler materials, metal-rich second phases are detected through detailed microprobe analyses.

The comparison of the thermoelectric properties of the differently prepared samples allows us to conclude that the microwave prepared TiNiSn sample performs at least as well as the conventionally prepared one while saving a significant amount of time and energy in the preparative process. In the case of the $\mathrm{TiCoSb}$ compounds, we find a metallic impurity phase, $\mathrm{CoSb}$, in the microwave prepared sample which results in significantly lower $z T$, when compared with conventionally prepared samples. The figure of merit of the $\mathrm{TiCoSb}$ compound in general is lower than that of TiNiSn and decomposition into $\mathrm{CoSb}$ below the optimum working temperature, which has been reported before, appears to be an impediment to further progress in this system.

\section{ASSOCIATED CONTENT}

\section{S Supporting Information}

Details of electron microprobe and hot-pressing conditions, SEM micrograph of TiNiSn, SEM micrographs obtained in backscattering mode, EDX analysis of the hot-pressed pellets, electronic properties obtained during three heating and cooling cycles. This material is available free of charge via the Internet at http://pubs.acs.org. 


\section{AUTHOR INFORMATION}

\section{Corresponding Author}

*E-mail: stucky@chem.ucsb.edu.

\section{Notes}

The authors declare no competing financial interest.

\section{ACKNOWLEDGMENTS}

C.S.B. is a recipient of the Feodor Lynen Research Fellowship supported by the Alexander von Humboldt foundation. W.G.Z thanks the Carl-Zeiss foundation and the Graduate School of Excellence MAINZ, supported by the state of Rheinland-Pfalz. J.E.D is supported by a fellowship from the ConvEne IGERT Program (NSF-DGE 0801627). B.R.L.is supported by an internship from the RISE program (NSF DMR 1121053). Y.Z. is a recipient of the Corning Fellowship, supported by the Corning Science Foundation. T.M.P. and R.S. are supported by the MRSEC Program (NSF DMR 1121053). G.D.S., C.E.M. and M.L.S. acknowledge support from the Center for Energy Efficient Materials (CEEM), an Energy Frontier Research Center funded by the U.S. Department of Energy, Office of Basic Energy Sciences under Award Number DESC0001009. Use of shared experimental facilities of the Materials Research Laboratory: an NSF MRSEC, supported by NSF DMR 1121053 is gratefully acknowledged. The MRL is a member of the NSF-supported Materials Research Facilities Network (www.mrfn.org). Use of the Advanced Photon Source at Argonne National Laboratory was supported by the U.S. Department of Energy, Office of Science, Office of Basic Energy Sciences, under Contract No. DE-AC02-06CH11357.

\section{REFERENCES}

(1) Sootsman, J. R.; Chung, D. Y.; Kanatzidis, M. G. Angew. Chem., Int. Ed. 2009, 48, 8616-39.

(2) Jeitschko, W. Metall. Trans. 1970, 1, 3159-3162.

(3) Pierre, J.; Skolozdra, R.; Tobola, J.; Kaprzyk, S.; Hordequin, C.; Kouacou, M.; Karla, I.; Currat, R.; Leliévre-Berna, E. J. Alloys Compd. 1997, 262-263, 101-107.

(4) Pierre, J.; Neel, L. J. Alloys Compd. 2000, 296, 243-252.

(5) Kandpal, H. C.; Felser, C.; Seshadri, R. J. Phys. D 2006, 39, 776785.

(6) Snyder, G. J.; Toberer, E. S. Nat. Mater. 2008, 7, 105-114.

(7) Kawaharada, Y.; Kurosaki, K.; Muta, H.; Uno, M.; Yamanaka, S. J. Alloys Compd. 2004, 384, 308-311.

(8) Xie, W.; Zhu, S.; Tang, X.; He, J.; Yan, Y.; Ponnambalam, V.; Zhang, Q.; Poon, S. J.; Tritt, T. J. Phys. D: Appl. Phys. 2009, 42, 235407.

(9) Qiu, P.; Huang, X.; Chen, X.; Chen, L. J. Appl. Phys. 2009, 106, 103703.

(10) Bhattacharya, S.; Pope, A. L.; Littleton, R. T., IV; Tritt, T. M.; Ponnambalam, V.; Xia, Y.; Poon, S. J. Appl. Phys. Lett. 2000, 77, 24762478.

(11) Bhattacharya, S.; Skove, M.; Russell, M.; Tritt, T.; Xia, Y.; Ponnambalam, V.; Poon, S.; Thadhani, N. Phys. Rev. B 2008, 77, 1-8.

(12) Bhattacharya, S.; Tritt, T. M.; Xia, Y.; Ponnambalam, V.; Poon, S. J.; Thadhani, N. Appl. Phys. Lett. 2002, 81, 43-45.

(13) Culp, S. R.; Poon, S. J.; Hickman, N.; Tritt, T. M.; Blumm, J. Appl. Phys. Lett. 2006, 88, 042106.

(14) Evers, C. B. H.; Richter, C. G.; Hartjes, K.; Jeitschko, W. J. Alloys Compd. 1997, 252, 93-97.

(15) Cook, B. A.; Harringa, J. L. J. Mater. Sci. 1999, 4, 323-327.

(16) Mastronardi, K.; Young, D.; Wang, C.; Khalifah, P.; Cava, R. J. Appl. Phys. Lett. 1999, 74, 8-10.

(17) Kim, S.-W.; Kimura, Y.; Mishima, Y. Intermetallics 2007, 15, 349-356.
(18) Aliev, F. G.; Kozyrkov, V. V.; Moshchalkov, V. V.; Scolozdra, R. V.; Durczewski, K. Z. Phys. D 1990, 357, 353-357.

(19) Baghurst, D.; Chippindale, A.; Mingos, D. Nature 1988, 332, 311.

(20) Roy, R.; Komarneni, S.; Yang, L. J. Am. Ceram. Soc. 1985, 68, $392-395$.

(21) Landry, C. C.; Barron, A. R. Science 1993, 260, 1653-1655.

(22) Whittaker, A. G.; Mingos, D. M. P. J. Chem. Soc., Dalton Trans. 1993, 2541-2543.

(23) Mastrovito, C.; Lekse, J.; Aitken, J. J. Solid State Chem. 2007, $180,3262-3270$.

(24) Agrawal, K. Curr. Opin. Solid State Mater. Sci. 1998, 3, 480-485.

(25) Xie, Z.; Wang, C.; Fan, X.; Huang, Y. Mater. Lett. 1999, 38, 190-196.

(26) Ramesh, P. D.; Brandon, D.; Scha, L. Mater. Sci. Engi., A 1999, 266, 211-220.

(27) Wong, W. L. E.; Karthik, S.; Gupta, M. Mater. Sci. Technol. 2005, 21, 1063-1070.

(28) Biswas, K.; Muir, S.; Subramanian, M. Mater. Res. Bull. 2011, 46, 2288-2290.

(29) Birkel, A.; Denault, K. A.; George, N. C.; Doll, C. E.; Hery, B.; Mikhailovsky, A. A.; Birkel, C. S.; Hong, B.-C.; Seshadri, R. Chem. Mater. 2012, 24, 1198-1204.

(30) Katz, J. D. Annu. Rev. Mater. Sci. 1992, 22, 153-170.

(31) Kimura, Y.; Ueno, H.; Mishima, Y. J. Electron. Mater. 2009, 38, 934-939.

(32) Baldinozzi, G.; Berar, J.-F.; Gautier-Soyer, M.; Calvarin, G. J. J. Phys. D.: Condens. Mater. 1997, 9, 9731-9744.

(33) Skovsen, I.; Bjerg, L.; Christensen, M.; Nishibori, E.; Balke, B.; Felser, C.; Iversen, B. B. Dalton Trans. 2010, 39, 10154-9.

(34) Katayama, T.; Kim, S. W.; Kimura, Y.; Mishima, Y. J. Electron. Mater. 2003, 32, 1160-1165.

(35) Zhou, M.; Chen, L.; Feng, C.; Wang, D.; Li, J.-F. J. Appl. Phys. 2007, 101, 113714

(36) Toberer, E. S.; Zevalkink, A.; Snyder, G. J. J. Mater. Chem. 2011, 15843-15852.

(37) May, A. F.; Toberer, E. S.; Saramat, A.; Snyder, G. J. Phys Rev. B 2009, 80, 125205.

(38) Tobola, J.; Pierre, J.; Kaprzyk, S.; Skolozdra, R.; Kouacou, M. J. Phys.: Condens. Matter. 1998, 1013. 